\title{
Higher-Order Associative Learning in Amnesia: Evidence from the Serial Reaction Time Task
}

\author{
Tim Curran \\ Case Western Reserve University
}

\begin{abstract}
Patients with anterograde amnesia are commonly believed to exhibit normal implicit learning. Research with the serial reaction time (SRT) task suggests that normal subjects can implicitly learn visuospatial sequences through a process that is sensitive to higher-order information that is more complex than pairwise associations between adjacent stimuli. The present research reexamined SRT learning in a group of amnesic patients with a design intended to specifically address the
\end{abstract}

\section{INTRODUCTION}

It is generally believed that implicit learning and memory are spared in patients with anterograde amnesia despite drastic impairments on standard tests of memory such as recognition and recall (for reviews see Moscovitch, Vriezen, \& Goshen-Gottstein, 1993; Schacter, Chiu, \& Ochsner, 1993). However, amnesic patients often show deficits on implicit tasks that require the formation of interitem associations (Bowers \& Schacter, 1993; Curran \& Schacter, in press). An apparent exception to the conclusion that amnesic patients show impaired associative implicit memory is provided by research on the serial reaction time (SRT) task (Nissen \& Bullemer, 1987; Nissen, Willingham, \& Hartman, 1989; Reber \& Squire, 1994). The present research examines the question of whether or not amnesic patients show normal associative learning in the SRT task by providing more stringent tests of learning higher-order information (i.e., information that is more complex than pairwise associations between temporally adjacent stimuli).

Graf and Schacter (1985) developed a paired-associate word-stem completion paradigm to study implicit memory for novel associations. Subjects studied nominally unrelated word pairs (e.g., knife-signal, peach-table) followed by a stem completion task in which three-letter stems were presented along with the previously paired associate (same context condition, peach-tab_) or a nonassociated word (different context condition, knifetab__. Graf and Schacter initially found that both amnesic and control subjects showed more priming in the learning of higher-order information. Despite seemingly normal learning effects on average, the results suggest that amnesic patients do not learn higher-order information as well as control subjects. These results suggest that amnesic patients have an associative learning impairment, even when learning is implicit, and that the medial temporal lobe and/or diencephalic brain areas typically damaged in cases of amnesia normally contribute to implicit sequence learning. same context condition than in the different one, so it was inferred the both groups learned novel associations. However, subsequent studies have produced conflicting and inconsistent results, with the weight of the evidence suggesting that amnesic patients do not show normal implicit memory for new associations (for review, see Bowers \& Schacter, 1993). Additional evidence suggests that amnesic patients can show normal implicit learning for new associations when such associations are incrementally acquired across multiple training trials (Musen \& Squire, 1993a, 1993b). SRT learning might also be interpreted as multiple-trial learning of new associations.

In the SRT task a visual stimulus is presented at one of four distinct spatial locations, and the subject presses a corresponding key as quickly and accurately as possible. Unbeknownst to subjects, the stimuli often follow a particular repeating pattern. For example, designating the four spatial locations as A to D from left to right, Nissen and Bullemer (1987) gave subjects continuous cycles of the sequence, D-B-C-A-C-B-D-C-B-A. Both amnesic Korsakoff's patients and control subjects were faster when stimuli followed the repeating sequence than when the stimulus locations were randomly determined, so Nissen and Bullemer inferred that both groups had learned the sequence. This finding was subsequently replicated and extended across a longer retention interval (Nissen et al., 1989), and related evidence has shown that normal subjects with scopolamine-induced amnesia show normal learning in this task (Nissen, Knopman, \& Schacter, 1987). SRT learning is often characterized as implicit when subjects fail to demonstrate explicit mem- 
ory of the sequence, although the evidence that learning is truly implicit continues to be debated (see Shanks \& St. John, 1994, and accompanying commentaries).

A closer look at the paradigm used by Nissen and colleagues has raised questions about the sequential and associative nature of SRT learning (Jackson \& Jackson, 1992; Reed \& Johnson, 1994; Shanks \& St. John, 1994). In Nissen and Bullemer's (1989) sequence (D-B-C-A-C-B-DC-B-A), some positions ( $B$ and $C$ ) are more frequent than others ( $A$ and $D$ ), so reaction times to sequence trials may be faster than reaction times to random trials because subjects learn something about relative frequency of occurrence. If subjects merely learn frequency information, SRT learning does not provide evidence for associative learning in amnesia.

A related issue concerns the extent to which sequence learning can be based on merely first-order associations between adjacent elements. In Nissen and Bullemer's (1989) sequence, RT could benefit from learning which first-order associations occur most frequently (Jackson \& Jackson, 1992; Reed \& Johnson, 1994). For example, $\mathrm{C}$ is followed by $\mathrm{B} 67 \%$ of the time, by $\mathrm{A} 33 \%$ of the time, and is never followed by $\mathrm{D}$. On average, reaction times would improve if subjects just learned that $\mathrm{B}$ was the most likely response to follow $\mathrm{C}$. Experiments with normal subjects (Cohen, Ivry, \& Keele, 1990; Reed \& Johnson, 1994; Stadler, 1993) have controlled for frequency learning and the learning of firstorder associations by creating random and sequence conditions in which each element occurs equally often (controlling for frequency learning) and each pairwise transition occurs equally often (controlling for pairwise learning).

Computational models of sequence learning have provided some insight into the nature of higher-order associative information that may be learned in the SRT task (Cleeremans, 1993; Cleeremans \& McClelland, 1991; Keele \& Jennings, 1992). These models rely on recurrent network architectures (e.g., Elman, 1990; Jordan, 1990) that allow each response to be predicted by a combination of previous stimuli. In these models the representation of a stimulus is influenced by the identify of previous stimuli, so in the sequence A-B-C-B-A-C, for example, the representation of the first $B$ is different from the representation of the second $B$ because they are preceded by different elements (A-B vs. C-B). Even though $B$ alone cannot uniquely predict the next location (C or $A$ ), $A-B$ uniquely predicts $C$, but $C-B$ predicts $A$. In the current terminology, this would be a form of second-order associative learning because the network learns not just pairwise (first-order) associations between immediately adjacent stimuli but uses information about two prior stimuli to predict the next. In fact, these networks develop even higher-order associations (thirdorder, fourth-order, etc.) because the representation of each stimulus is a function of all previous stimuli, with more remote stimuli having a lesser impact than nearby stimuli. Some computational and behavioral evidence has converged on the estimate that the prediction of each element is significantly influenced by the identity of at least three prior elements (Cleeremans \& McClelland, 1991).

Although research with normal subjects and network models of sequence learning have promoted the idea that SRT learning generally depends on higher-order information, it remains possible that amnesic patients relied on frequency learning or pairwise probability learning in experiments that used Nissen and Bullemer's (1989) sequence. Some of these concerns have recently been addressed by Reber and Squire (1994). First, the paper provided the clearest evidence to date that amnesic patients showed normal sequence learning but did not exhibit significant explicit knowledge. Second, and most important for the present purposes, Reber and Squire's Experiment 2 used a sequence that could not be completely learned on the basis of first-order associations or relative frequency of the sequence elements. A 12-item sequence was used in which each of four positions was repeated three times, and each position was equally likely to be followed by each other position (e.g., B-C-D-B-A-D-A-C-A-B-D-C). The sequence cannot be learned on the basis of frequency information because each element appears equally often. The sequence cannot be learned completely from first-order associations because all first-order transitions (e.g., A-B, A-C, A-D; B-A, B-C, B-D; C-A, C-B, C-D; D-A, D-B, D-C) are equally likely. The random control condition also, on average, should have equally probable locations and pairwise transitions (except immediate repetitions), so any reaction time difference between random and sequence conditions should be attributable to learning of higher-order information. Reber and Squire found that learning of this sequence was not significantly different between control subjects and amnesic patients. Thus, amnesic patients appeared to learn higher-order associations in the SRT task as well as control subjects. However, although the learning by group interaction was not statistically significant, the control subjects showed a learning effect (random vs. sequence) that was numerically larger than the amnesic patients' effect (Reber \& Squire, 1994, Figure 3). In addition, Nissen and Bullemer found a significant group by learning interaction that indicated greater learning by control than amnesic subjects. The present research sought to reevaluate the learning of higher-order associations with design modifications intended to more clearly evaluate higher-order associative learning.

First, the sequence learning ability of amnesic patients was tested with two sequences that differed in the predictiveness of pairwise associations. The first-order predictive (FOP) sequence included pairwise information that was probabilistically predictive (A-B-A-D-B-C-DC-A-D-B-C). That is, each element (e.g., A) is followed by one element $67 \%$ of the time (D) and another element 
$33 \%$ of the time (B) but is never followed by the third element. Normal subjects can learn such pairwise probabilities in the SRT task Jackson \& Jackson, 1992; Stadler, 1992), and amnesic patients have shown normal learning of pairwise probabilities in a probabilistic classification task (Knowlton, Squire, \& Gluck, 1994). In the second-order predictive (SOP) sequence (A-B-A-D-B-C-D-A-C-B-D-C) each stimulus is equally likely to be followed by any other stimulus, so pairwise information is inadequate for learning. This sequence is similar to the sequence in Reber and Squire's (1994) Experiment 2. Each stimulus can only be predicted based on some combination of previous stimuli. For example, although $B$ does not uniquely predict its successor (B-A, B-C, B-D are equally likely), the combination of $B$ plus the previous stimulus does uniquely predict the next location ([A-B]-A, [D-B]-C, [C-B]-D). Thus, learning of the SOP sequence depends on second-order, or higher-order, associations.

Secondly, the present experiment departed from the standard blocked arrangement of random and sequence conditions that has been used in all past studies of SRT learning with amnesic patients (Nissen \& Bullemer, 1987; Nissen et al., 1989; Reber \& Squire, 1994). In past studies, subjects completed four or five consecutive blocks of trials with the sequence followed by a fifth random block, and learning was assessed as the reaction time difference between the last sequence block and the random block. The present design (following Stadler, 1993) included intermixed cycles of random and sequence trials within each block. Using $R$ to denote 12 random trials and $S$ to denote one cycle of the 12-element sequence (e.g., SOP: A-B-A-D-B-C-D-A-C-B-D-C), each block of 120 trials was arranged as R-S-S-R-S-S-R-S-S-R. This design has the advantage of obscuring the presence of the sequence, so subjects are less likely to explicitly recognize it. It also allows a within-subject measure of learning to be obtained in each block rather than merely assessing learning at a single point toward the end of the experiment.

The intermixing of random and sequence cycles also has an advantage specifically related to the assessment of higher-order associative learning. Even if overall RTs differ between the random and sequence blocks for the SOP sequence, it may be possible that subjects merely learned isolated pairs within the sequence. If, by chance, learned pairs occurred more often in sequence trials than random ones, average reaction times would be faster on the sequence trials. Learning can be assessed while controlling for this pairwise information by comparing the reaction time for each pairwise transition between the random and sequence conditions. For example, subjects might learn the A-B pair in a sequence. If subjects only learn such pairs, RT to $B$ when preceded by $A$ would be equal for random and sequence conditions. If RT to $A-B$ is faster for sequence trials than random ones, subjects must have learned some higherorder information from the sequence that allows them to respond to $A-B$ faster in the sequence condition than in the random one. This method of explicitly comparing reaction times to pairwise elements within the random and sequence conditions would have been impossible in past experiments because of an insufficient number of random trials to obtain stable $\mathrm{RT}$ estimates for each pairwise transition.

In summary, the present experiment sought to investigate higher-order associative learning in amnesic patients with a modified SRT task. Each amnesic subject was tested with two sequences: one with predictive pairwise associations (FOP, A-B-A-D-B-C-D-C-A-D-B-C) and the other with nonpredictive pairwise associations but predictive second-order associations (SOP, A-B-A-D-B-C-DA-C-B-D-C). Amnesic patients were tested in two sessions that were at least 3 months apart, but control subjects (two matched with each amnesic patient) were only tested on a single sequence because they were likely to remember the debriefing after the first session.

\section{RESULTS}

Sequence type (FOP vs. SOP) was treated as a repeated measure in all statistical comparisons. This designation is obvious for amnesic patients who were tested on both sequences. Although control subjects only were tested on one sequence, they were matched on the basis of age and education, so repeated-measures statistics were appropriate.

Though reaction time (RT) is the dependent measure of primary interest, it is important to establish that RT analyses are not compromised by speed-accuracy tradeoffs. A group (amnesic vs. control) $\times$ sequence (FOP vs. SOP) $\times$ learning (random vs. sequence) $\times$ block (1-9) ANOVA was computed from the mean percentage correct. Control subjects $(97.6 \%)$ were marginally more accurate than amnesic patients $(95.5 \%), F(1,18)=4.12$, $M S E=194.33, p=0.06$. Subjects were significantly more accurate on sequence trials $(97.1 \%)$ than on random trials $(96.1 \%), F(1,18)=16.46, M S E=10.63, p<0.001$; and the size of this learning effect increased across blocks, $F(8,144)=2.87, M S E=9.22, p<0.01$. Overall accuracy decreased across blocks from a high of $97.4 \%$ in Block 1 to a low of $95.6 \%$ in Block $8, F(8,152)=2.24$, $M S E=11.17, p<0.05$. No other effects approached statistical significance. As confirmed below, with only one exception, accuracy effects were consistent with RT effects (e.g., sequence trials were both faster and more accurate than random trials). The one exception was a speed-accuracy trade-off across blocks-subjects became faster but less accurate across blocks. Because the main effect of blocks was of little interest, this trade-off did not undermine interpretation of the RT data.

Only RTs from trials that were correct and less than 1200 msec were included in the analyses. A group (amnesic vs. control) $\times$ sequence (FOP vs. SOP) $\times$ learning (random vs. sequence) $\times$ block (1-9) ANOVA was com- 
puted from subjects' median RTs in each condition. Although block was entered as a nine-level factor in the ANOVA, Figure 1 displays the average of each contiguous set of three blocks $(1-3,4-6,7-9)$ in order to simplify the display. The learning effect was highly significant, $F(1$, 19) $=20.13, M S E=2990.59, p<0.001$. Subjects became faster across blocks, $F(8,144)=7.88, M S E=2180.92$, $p<0.001$, and learning increased across blocks, $F(8$, $144)=2.11, M S E=541.83, p<0.05$. This learning by block interaction is not readily apparent in Figure 1 because it primarily occurred across the first three blocks that are averaged together. Learning also inter- acted with the sequence type, $F(1,18)=6.44, M S E=$ $1096.35, p<0.05$. This interaction reflects greater learning of the SOP than the FOP sequence, but planned comparisons (two-tailed $t$ tests on RTs averaged over blocks) confirmed that the learning effect was significant for each sequence individually: FOP (random $=549$, sequence $=537), t(19)=3.01, S E=5.23, p<0.01 ; \mathrm{SOP}$ $($ random $=556$, sequence $=532), t(19)=4.70, S E=5.23$, $p<0.001$. Most importantly, there were no significant interactions with group.

It is crucial that no group differences in overall RT were obtained, $F<1$. Past studies have found that amne-
Figure 1. Reaction time (means of subjects' medians) for random practice blocks and the average of each three consecutive experimental blocks. Reaction time for amnesic patients and control subjects to random and sequential trials are plotted separately. (A) First-order predictive sequence (FOP). (B) Second-order predictive sequence (SOP).

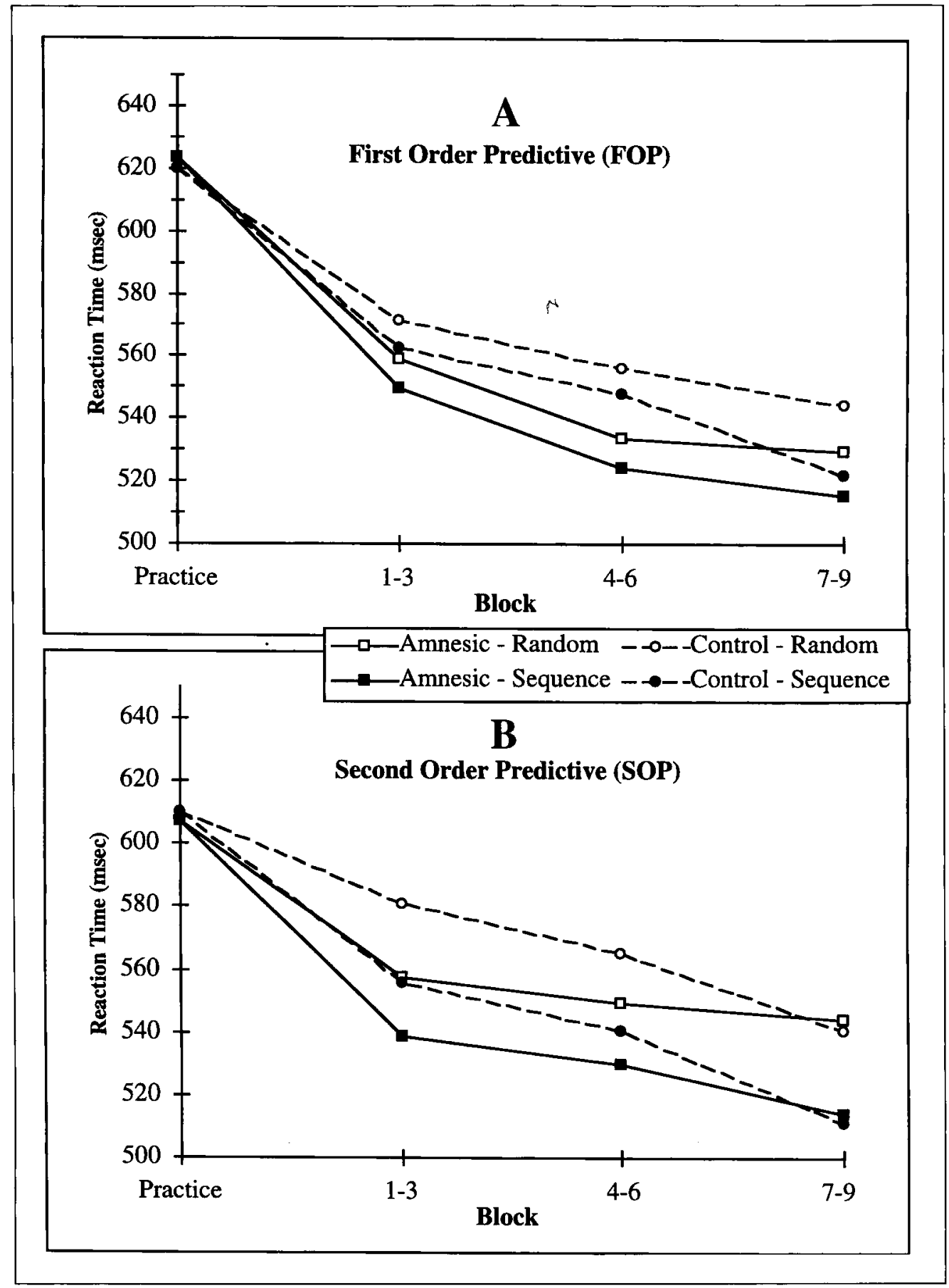


sic patients were significantly slower than control subjects (Nissen \& Bullemer, 1987; Nissen et al., 1989). Such baseline RT differences can obscure potential differences in learning between the groups. Learning effects are based on the difference between random and sequence trials, and RT differences are known to increase with overall RT (Chapman, Chapman, Curran, \& Miller, 1994). Therefore, the learning effects shown by amnesic patients could have been artifactually inflated if amnesic patients were slower than control subjects.

Despite the absence of significant group effects in the ANOVA, it is important to confirm that the amnesic group alone learned each of the sequences. As observed for both groups combined, amnesic subjects alone showed a significant learning effect, $F(1,9)=8.84, M S E=$ $2865.06, p<0.05$, but the learning by sequence interaction was not significant, $F(1,9)=2.55, M S E=1218.54$, $p=0.14$. Planned comparisons revealed that amnesic subjects showed significant learning of each sequence: FOP $($ random $=541$, sequence $=530), t(9)=2.49, S E=$ $4.37, p<0.05$; SOP (random $=550$, sequence $=528$ ), $t(9)=2.38, S E=9.53, p<0.05$. The results were qualitatively similar for control subjects, except that the learning by sequence interaction was marginally significant, $F(1,9)=4.09, M S E=974, p=0.07$. Overall, the results suggest that amnesic patients learned both the FOP and the SOP sequence as well as control subjects. However, the amnesic patients, unlike the control subjects, did not show a larger learning effect for the SOP than for the FOP sequence. The next analysis suggests that amnesic patients may not have learned the SOP sequence better than the FOP sequence because of a deficit in learning higher-order information.

To specifically examine whether subjects learned more than pairwise information, RTs to each pairwise transition were compared between the random and sequence conditions. Figure 2 plots the difference between RTs to each sequence element versus average RTs to random trials that occurred in the same pairwise combination. For example, the rightmost points in Figure $2 \mathrm{~A}$ correspond to the difference between RT to $\mathrm{C}$ when preceded by $\mathrm{B}$ in random trials (B-C) versus $\mathrm{C}$ as the last element of the FOP sequence. It can be seen that both groups were about $30 \mathrm{msec}$ faster with $\mathrm{B}-C$ when it occurred at the end of the FOP sequence than when B- $C$ occurred within random trials. Difference scores that are significantly above zero provide clear evidence of higher-order associative learning because all effects of individual and pairwise items are controlled.

For each subject, mean reaction times (across all blocks) were computed for each sequence element and each occurrence of the corresponding pairwise combination in random trials. These means were analyzed in group (amnesics vs. control) by learning (random vs. sequence) by position (1-12) ANOVAs separately for each sequence type. For the FOP sequence, only the effects of position, $F(11,198)=2.35, M S E=5028, p<$
0.01 , and the learning by position interaction, $F(11$, 198) $=5.96, M S E=1323, p<0.001$, were significant. No group effects approached significance. The nonsignificant effect of learning, $F(1,18)=1.17, M S E=2576$, suggest that higher-order sequence information was not learned across all positions, but the learning by position interaction suggests higher-order learning of some individual positions.

Separate planned comparisons (two-tailed $t$ tests) for each group tested the significance of the random vs. sequence learning difference for each position. The results of these $t$ tests are indicated in Figure 2A (Controls: ${ }^{*} p<0.05$; Amnesics: ${ }^{+} p<0.05$ ). First, each group showed only a single position on which sequence RTs were significantly faster than random RTs to the corresponding pair. Both groups also showed negative differences that attained significance, indicating faster RTs to pairs within random than within sequence conditions. These negative differences will be discussed later, but the primary result of this analysis is that little higher-order information was learned by either group.

For the SOP sequence (Figure 2B), the ANOVA indicated a significant difference between random and sequence conditions, $F(1,18)=15.14, M S E=3486, p<$ 0.01 . This learning effect interacted with position, $F(11$, 198) $=6.725, M S E=838, p<0.001$, and the main effect of position was also significant, $F(11,198)=2.71, M S E=$ $6328, p<0.01$. Thus, there was significant learning of higher-order information across all positions (on average), but the amount of higher-order learning varied between positions. Although no group effects approached significance, additional ANOVAs were performed on each group separately in order to ensure that each group learned more than pairwise information. Control subjects showed a significant difference between random and sequence, $F(1,9)=13.34, M S E=$ $2749, p<0.01$, and this difference interacted across positions, $F(11,99)=4.62, M S E=762, p<0.001$. For amnesic patients the learning effect only approached significance, $F(1,9)=4.21, M S E=4222, p=0.07$, but interacted with position, $F(11,99)=2.98, M S E=914$, $p<0.01$. Thus, control subjects learned more than pairwise information across all positions, but higher-order learning of amnesic patients was only marginally significant. Planned comparisons (two-tailed $t$ tests) were again used to examine learning for each position and each group separately (Figure 2B; Controls: ${ }^{*} p<0.05$, ${ }^{* *} p<0.01$; Amnesics: ${ }^{+} p<0.05$ ). Figure $2 \mathrm{~B}$ suggests that control subjects learned high-order information about more elements of the SOP sequence than did amnesic patients. This is consistent with the ANOVA, which showed learning by amnesic patients to be only marginal across all positions.

As shown in Figure 2B, amnesic patients showed significant learning of SOP positions 1,2 , and 10. Inspection of individual subjects' differences revealed that the same subjects were learning these three positions. The corre- 
Figure 2. Differences between mean reaction times to each sequence element and reaction times to the same pairwise combinations in random trials. Reaction times were averaged across all blocks of trials. Positive differences reflect learning of higher-order sequence information. Error bars represent the standard error of the mean. $T$ tests (twotailed, $d f=9$ ) were used to assess the significance of the learning effect for each group and each sequence position separately (control subjects: ${ }^{*} p<0.05,{ }^{* *} p<0.01$; amnesic patients: ${ }^{+} p<0.05$ ). (A) First-order predictive sequence (FOP). (B) Secondorder predictive sequence (SOP).



lations between the amnesic patients' learning differences at each of these three positions were highly positive ( 1 vs. $2: r=0.80,2$ vs. $10: r=0.87,1$ vs. $10: r=0.79$, all $p<0.01$ ). When the average difference was calculated across these three positions, four amnesic patients showed learning differences that were greater than two standard errors from zero ( $S E=17 \mathrm{msec}$ ). Unfortunately, there is nothing known to be common about these four subjects (DF, PS, LB, PD, see Table 2) that might explain their better learning. They had amnesia attributable to diverse etiologies, and learning was uncorrelated with age or WMS-R performance.

Explicit knowledge of the sequence was assessed by a multiple choice question and two recognition tests.
The top of Table 1 presents the number of subjects who gave each possible answer to the question: "The movement of the black box is best described as ?" "Random" was selected more often than the other alternatives for both amnesic and control subjects. Thus, it appears that most subjects did not notice the repeating sequence. A more objective assessment of subjects' explicit knowledge was made with the fragment and whole sequence recognition results that are shown at the bottom of Table 1. Recognition scores were computed for each subject on each test by taking the average rating to targets and subtracting the average rating to distractors. A score of 100 indicates perfect discrimination between targets and distractors, and a score of 0 indicates chance perfor- 
Table 1. Results of Explicit Knowledge Question and Recognition Tests.

\begin{tabular}{|c|c|c|c|c|}
\hline \multirow[b]{3}{*}{ Explicit Knowledge Questions } & \multicolumn{4}{|c|}{ No. of Subjects Who Gave Each Response } \\
\hline & \multicolumn{2}{|c|}{ Amnesic Patients } & \multicolumn{2}{|c|}{ Control Subjects } \\
\hline & $F O P$ & $S O P$ & FOP & SOP \\
\hline \multicolumn{5}{|l|}{ The movement of the black box is best described as? } \\
\hline Random & 10 & 6 & 3 & 7 \\
\hline Some positions occurred more often than others & & 1 & 3 & 3 \\
\hline The movement was often predictable & & 2 & 3 & \\
\hline The same sequence of movements would often appear & & & 1 & \\
\hline $\begin{array}{l}\text { The same sequence of movements occurred throughout } \\
\text { the experiment }\end{array}$ & & 1 & & \\
\hline \multicolumn{5}{|l|}{ Mean recognition scores (target ratings - distractor ratings) } \\
\hline Whole sequence recognition & 2.57 & 0.36 & 3.40 & -14.86 \\
\hline Fragment recognition & 5.08 & -0.25 & -0.27 & -0.33 \\
\hline Mean recognition & 3.83 & 0.05 & -1.57 & -7.60 \\
\hline
\end{tabular}

mance. Neither group showed above-chance discrimination between targets and distractors in either the whole sequence or fragment recognition tests. Separate group by sequence type ANOVAs for each recognition test revealed no effects approaching significance. The sensitivity of these tests to explicit knowledge, when it exists, has been confirmed in an experiment with undergraduates in which both whole sequence and fragment recognition were significantly better when subjects were told there was a sequence before the SRT task than when they were not told about the sequence (Curran, 1997). Thus, these recognition tests are valid measures of explicit memory (see also Perruchet \& Amorim, 1992; Willingham, Greenley, \& Bardona, 1993). The low levels of explicit knowledge in this experiment are probably attributable to the intermixing of random and sequence trails within each block.

\section{DISCUSSION}

Amnesic patients and control subjects showed very similar (and not statistically different) sequence learning when it was assessed as the overall RT difference between sequence and random conditions (Figure 1). Amnesic patients appeared to show normal learning of a sequence with predictive pairwise information (FOP, Figure $1 \mathrm{~A}$ ) as well as a sequence that demanded learning of at least second-order associations (SOP, Figure 1B). However, control subjects learned the SOP sequence better than the FOP sequence, but amnesic subjects showed similar amounts of learning for both sequences. When higher-order learning was examined directly by comparing RT to each pairwise transition in random versus sequence conditions, amnesic patients exhibited some deficit (Figure 2). Neither group showed convincing evidence for higher-order learning of the FOP sequence (Figure 2A), and controls showed more complete higher-order learning of the SOP sequence than the amnesic patients (Figure 2B). These results suggest that amnesic patients' ability to learn higher-order associations was impaired.

The finding that the second-order predictive (SOP) sequence was better learned than the first-order predictive (FOP) sequence may appear surprising, but there are two likely explanations for this result. First, pairwise probability learning would result in imperfect sequence knowledge. For example, if subjects learn that $A$ is most likely to be followed by $D$, this would only help them on two-thirds of the trials that follow A. In fact, there is some evidence that this sort of knowledge may have hurt subjects on trials where such expectancies were violated. To wit, all of the significantly negative scores in Figure $2 \mathrm{~A}$ represent responses to less probable transitions (A-B is less likely than A-D, B-A is less likely than $B-C$, and D-C is less likely than D-B). Thus, there may be a cost associated with pairwise probability learning. Because this explanation depends on results of the analysis of pairwise differences between random and sequence conditions (Figure 2A), it requires some sort of higherorder knowledge that would make these less likely transitions slower in the sequence than random conditions, so the exact explanation of these effects is uncertain.

A second reason for poor learning of the FOP sequence is a repeating subsequence that may have complicated learning (A-D-B-C, underscored in Figure 2A). The presence of this subsequence necessitates some sensitivity to remote contingencies in order to learn other aspects of the sequence. That is, the subject must 
Table 2. Characteristics of Amnesic Patients

\begin{tabular}{|c|c|c|c|c|c|c|}
\hline \multirow[b]{2}{*}{ Patient } & \multirow[b]{2}{*}{ Etiolog $y^{a}$} & \multirow[b]{2}{*}{ Age } & \multirow[b]{2}{*}{$V I Q^{a}$} & \multicolumn{3}{|c|}{ Wecbsler Memory Scale-Revised ${ }^{a}$} \\
\hline & & & & $G M$ & $A T N$ & $D L Y$ \\
\hline ds & Anoxia & 31 & 95 & 65 & 120 & $<50$ \\
\hline ps & Anoxia & 36 & 104 & 90 & 115 & $<50$ \\
\hline pd & Anoxia & 57 & 115 & 65 & 89 & 61 \\
\hline ss & Enceph & 67 & 126 & 102 & 114 & $<50$ \\
\hline $\mathrm{df}$ & Enceph & 44 & 111 & 81 & 107 & 69 \\
\hline $\mathbf{c w}$ & Stroke & 53 & 84 & 79 & 89 & 80 \\
\hline gp & Kors & 73 & 123 & 104 & 116 & 56 \\
\hline $\mathrm{pb}$ & Kors & 68 & 87 & 82 & 93 & 60 \\
\hline wr & Kors & 66 & 88 & 76 & 96 & 53 \\
\hline \multirow[t]{2}{*}{ lb } & Kors & 60 & 87 & 99 & 99 & 61 \\
\hline & Mean & 56 & 102 & 84 & 104 & 59 \\
\hline \multirow[t]{2}{*}{ Norms: } & Mean & & 104 & 99 & 100 & 101 \\
\hline & Stdev & & 13 & 14 & ริ5 & 15 \\
\hline
\end{tabular}

${ }^{a} \mathrm{VIQ}=$ Verbal IQ, GM = General Memory Subscale, $\mathrm{ATN}=$ Attention Subscale, DLY = Delayed Memory Subscale, Enceph $=$ encephalitis, Kors $=$ Korsakoff's disease

use the element which immediately precedes the subsequence in order to correctly predict the successor of the subsequence (B-A-D-B-C-D vs. $C-\mathrm{A}-\mathrm{D}-\mathrm{B}-\mathrm{C}-A$; i.e., fifthorder associations). Not only is prediction of the successor to this subsequence (D vs. A) dependent on the identity of its predecessor (B vs. C) but so is prediction of the interior members of the subsequence $(D, B, C)$. These ambiguities may have complicated higher-order learning of the FOP sequence, and the learning effects in Figure 1A may primarily reflect pairwise learning. This interpretation is supported by the observation that little higher-order learning was evident in the analysis of pairwise differences (Figure 2A).

In contrast to the FOP sequence, the SOP sequence is completely learnable by second-order associations because each pair uniquely predicts the next stimulus/ response: $[A-B]-A,[B-A]-D,[A-D]-B$, etc. For the SOP sequence, there was only one isolated case where learning of pairwise associations was significantly negative (Figure $2 \mathrm{~B}$, last position). This is consistent with the idea that the negative pairwise differences in Figure $2 \mathrm{~A}$ are attributable to overreliance on first-order probabilities, because first-order probabilities are completely nonpredictive in the SOP sequence. It is apparent from Figure 2B that control subjects showed superior higher-order learning compared to the amnesic patients-despite the appearance of normal learning when average RTs were compared (Figure 1B). It seems possible that amnesic subjects were somewhat able to learn second-order associations (some positions were learned as seen in Fig- ure 2B), but they may not have been able to learn as many of these associations as control subjects. Alternatively, control subjects, but not amnesic patients, may have learned sequential information that is higher than second-order. Some evidence for this might be seen in the three contiguous positions in the middle of the SOP sequence that were learned by control subjects.

The present results question standard neuropsychological views of SRT learning (in particular) and implicit skill learning (in general). As reviewed in the introduction, SRT learning has been claimed to be normal in amnesic patients (Nissen et al., 1989; Reber \& Squire, 1994), even though Korsakoff's patients learned less than control subjects in Nissen and Bullemer's (1987) original study - possibly because control subjects had more explicit knowledge. Previous studies of SRT learning in amnesic patients (Nissen \& Bullemer, 1987; Nissen et al., 1989; Reber \& Squire, 1994, Experiment 1) have used a sequence (D-B-C-A-C-B-D-C-B-A) in which relative frequency and predictive first-order probabilities could have been learned (Jackson \& Jackson, 1992; Reed \& Johnson, 1994; Shanks \& St. John, 1994), so the content of the learned information is difficult to infer. These sequence characteristics may account for the seemingly normal learning of amnesic patients in these experiments.

Reber and Squire's (1994) Experiment 2 overcame these limitations by using a SOP sequence, like that used in the present experiment. Subjects were trained on four sequential blocks and then they were given two tests of 
explicit knowledge before they completed another sequential block and then a random block. They considered two measures of learning: the decrease in reaction time across the first four-sequence blocks and the reaction time difference between the final sequence and random blocks. A main effect of decreasing RTs across the first four blocks along with a nonsignificant block by group interaction was used to infer normal learning by the amnesic subjects. Normal learning was also inferred from a significant difference between the final random and sequence blocks that did not interact with the group and was significant for each group separately. These results are consistent with the present analysis of average reaction times (Figure 1). However, the present finding that amnesic patients showed impaired higher-order learning (Figure 2) may explain why Reber and Squire's patients showed a learning difference that was numerically less than control subjects' difference (though their learning by group interaction was not statistically significant).

Another important difference between these experiments concerns the use of intermixed random and sequence cycles in the present experiment rather than uninterrupted sequence training (Reber \& Squire, 1994). It is possible that the intermixed random trials had a more disruptive effect on learning by patients with amnesia than on control subjects. It has been suggested that amnesic patients show a greater susceptibility to such forms of interference (e.g., Shapiro \& Olton, 1994), so this issue warrants further investigation.

Not only do the present conclusions depart from previous SRT research with amnesic patients, they also may seem inconsistent with other evidence suggesting that the basal ganglia and motor cortical areas (primary motor cortex, supplementary motor areas, premotor areas) play a prominent role in SRT learning (for review, see Curran, 1995). For example, the basal ganglia has been implicated by findings of SRT learning deficits in patients with Huntington's disease (Willingham \& Koroshetz, 1993) and Parkinson's disease (Jackson, Jackson, Harrison, Henderson, \& Kennard, 1995), and nuclei of the basal ganglia have shown learning-related activity in PET studies of SRT learning (Grafton, Hazeltine, \& Ivry, 1995; Rauch et al., 1995). However, the exact contribution of the basal ganglia to sequence learning remains elusive (Curran, 1995; Helmuth \& Ivry, in press), and there is no reason to suspect that the basal ganglia is the only brain area that contributes to SRT learning.

Neuroimaging studies implicating motor cortical areas (Grafton et al., 1995; Pascual-Leon, Grafman, \& Hallet, 1994; Rauch et al., 1995) also cannot rule out the contribution of other brain areas. Indeed, PET studies of SRT learning have found significant learning-related activity in areas damaged in amnesic patients, although the authors have not emphasized these findings. Rauch et al. found that the thalamus was more active in sequence than random conditions, but limited spatial resolution does not allow for localization within the nuclei that are spe- cifically damaged in amnesic patients. Grafton et al. found that hippocampal activity decreased with learning-a finding that is entirely consistent with other evidence that hippocampal activity is related to the novelty of a stimulus (Stern et al., 1996; Tulving, Markowitsch, Kapur, Habib, \& Houle, 1994). It is notable that previous neuropsychological and neuroimaging studies of SRT learning have provided very little information about the function that the implicated brain areas contribute to sequence learning. From past studies we can merely infer that the basal ganglia and motor cortical areas are somehow involved in sequence learning (Curran, 1995). The present study has specifically isolated the learning deficit of amnesic patients to higher-order associative learning.

The present results are surprising from the common perspective that amnesic patients show normal learning on tasks that might be characterized as "skill learning," "procedural learning," or "implicit learning." SRT learning is often considered to be an example of procedural or skill learning (e.g., Gabrieli, 1994; Squire, 1992), but such a classification may be imprecise. If skill learning represents learning "how" (e.g. Cohen \& Squire, 1980) or learning general cognitive procedures, rather than learning specific information, "skill learning" is likely to be a misleading descriptor for the kind of information that is acquired in the SRT task (others have expressed similar concerns about the classification of SRT learning, Moscovitch et al., 1993). General procedures or skills may be learned in the SRT task-as indicated by reaction time decrease across random trials (Figure 1). However, when SRT learning is measured as the $R$ T difference between performance on random versus sequence conditions, learning is specific to the practiced sequence and does not reflect nonspecific learning of a skill.

In agreement with the present finding of impaired implicit learning of higher-order associations, other research indicates implicit memory is not entirely preserved in amnesia when the task requires associative learning (Curran \& Schacter, in press). Characterization of the functional deficits that underlie amnesia is likely to be more successful when focused on underlying processes (i.e., associative learning) rather than on superficial task characteristics (see Mayes \& Downes, in press, and accompanying commentaries). Finally, it is notable that the present findings are generally consistent with theories of hippocampal and/or medial temporal lobe function that emphasize the role of these brain areas in learning higher-order associations between multiple stimuli (e.g., Cohen \& Eichenbaum, 1993; Gluck \& Myers, 1992; Rudy \& Sutherland, 1994; Wicklegren, 1979).

\section{METHOD}

\section{Subjects}

Subjects were 10 amnesic patients and 20 matched controls. An eleventh amnesic patient and his two control 
subjects were tested but discarded because the patient's etiology was atypical compared to the others (aneurysm of the anterior communicating artery ${ }^{1}$ ). The amnesic patients were obtained through the Memory Disorders Research Center of the Boston VA Medical Center. Amnesia was attributable to alcoholic Korsakoff's syndrome in four patients, and the remaining six patients had different etiologies [encephalitis (2), anoxia (3), and thalamic infarct (1)]. Each patient's age, verbal IQ, and Wechsler Memory Scale (WMS-R) scores are listed in Table 2. Each amnesic subject was paired with two age- and educationmatched control subjects. Control subjects for the Korsakoff's patients were nondrinking alcoholics.

\section{Stimuli and Apparatus}

Stimulus presentation and response selection were controlled by a Macintosh computer. Stimuli were four rectangular boxes $(35 \times 21 \mathrm{~mm}, 8 \mathrm{~mm}$ between boxes) that were centered on the computer monitor. Response keys were the $\mathrm{C}, \mathrm{V}, \mathrm{B}$, and $\mathrm{N}$ keys of the Macintosh keyboard, and subjects used the first two fingers of each hand to respond.

\section{Design}

Each amnesic subject participated in two experimental sessions that were separated by at least 3 months. The subject was given the first-order predictive (FOP, A-B-AD-B-C-D-C-A-D-B-C) sequence in one session and the second-order predictive (SOP, A-B-A-D-B-C-D-A-C-B-D-C) sequence in the other. Order of these sequence types was counterbalanced across subjects so that half the subjects were given the FOP sequence first, and vice versa for the other subjects. Because subjects were debriefed about the repeating sequence before completing the recognition measures, control subjects would presumably remember the debriefing across sessions. Therefore, each control subject was only tested on a single sequence, and each amnesic patient had two matched controls, one for each session. Sequence elements (A, B, C, D) were assigned to different spatial locations $(1,2,3$, 4) across subjects. For three subjects: $A=1, B=2, C=$ $3, \mathrm{D}=4$; for three subjects: $\mathrm{A}=2, \mathrm{~B}=3, \mathrm{C}=4, \mathrm{D}=1$; for four subjects: $A=3, B=4, C=1, D=2$.

\section{Procedure}

Subjects were told that the experiment was simply concerned with speed, accuracy, and stamina. The presence of a repeating sequence was not mentioned until after the serial reaction time (SRT) task was completed. Subjects were told that a different box would be filled on each trial, and they should press the corresponding key as quickly and accurately as possible. Stimuli remained on the screen until a response was given, and the next stimulus appeared $200 \mathrm{msec}$ after each response.
The first block of each session was 120 random practice trials. In random conditions, the stimulus location was randomly selected on each trial with the constraint that no location was immediately repeated. Next, subjects were given nine more blocks of trials with intermixed cycles of random $(R=12$ random trials) and sequence ( $\mathrm{S}=$ one sequence cycle) conditions arranged as: R-S-S-R-S-S-R-S-S-R. Each sequence cycle began in a random position within the sequence.

After the SRT task, subjects were asked if the stimulus movement was best described as (1) random, (2) some positions occurred more often than others, 3) the movement was often predictable, (4) the same sequence of movements would often appear, or (5) the same sequence of movements occurred throughout the entire experiment. More objective measures of explicit knowledge were obtained in two sequence recognition tasks.

First, in the whole sequence test (Willingham et al. 1993), subjects were asked to discriminate between the practiced sequence and seven distractor sequences. Sequences were presented as numbers (e.g., 1-2-1-4-23-4-1-3-2-4-3). Eight different sequences were constructed by taking the two sequences (FOP and SOP) and making four forms of each by assigning each location number (1-4) to each sequence position letter (AD). Thus, all subjects saw the same test choices, but target sequence differed between subjects. Subjects rated each sequence on a 100-point scale according to their certainty that the sequence was repeatedly presented in the experiment $(0=$ certain it was not repeated; $100=$ certain it was repeated).

Second, in the fragment recognition test (Perruchet $\&$ Amorim, 1992) subjects chose between 12 different fourelement subsequences (or fragments). Half of these fragments were taken from the studied sequence, and half were taken from the other sequence type. Thus, if the subject was given the FOP sequence in the SRT task, she or he was required to discriminate between four-element fragments of the practiced FOP sequence and the nonpracticed SOP sequence. As in the whole sequence recognition test, subjects rated each sequence on a 100point scale. Recognition scores were obtained for each test by taking the average rating to targets and subtracting the average rating to distractors.

\section{Acknowledgments}

This research was conducted while the author was a postdoctoral fellow in Daniel L. Schacter's laboratory at Harvard University. This project was supported by Program Project Grant NS 26985 from the National Institute on Neurological Disorders and Stroke to Boston University. Preparation of this paper was partly supported by a W. P. Jones Presidential Faculty Development Award from Case Western Reserve University. I thank Elisa Bolton for testing subjects, Dan Schacter for helpful advice throughout this research, Mieke Verfaellie for providing information on patients' backgrounds, and the Memory Disorders Research Center of the Boston VA Medical Center for allowing me to work with the amnesic patients. I also thank 
Allison Marks and Russell Poldrack for commenting on an earlier draft of this paper.

Reprint requests should be sent to Tim Curran, Department of Psychology, Case Western Reserve University, Cleveland, $\mathrm{OH}$ 44106-7123. E-mail: tec3@po.cwru.edu.

\section{Notes}

1. Anterior communicating artery aneurysms often produce a form of amnesia that is qualitatively different from the global amnesia that is typically attributable to medial temporal or diencephalic lesions (Parkin \& Leng, 1993). This subject's data were discarded prior to analyses.

\section{REFERENCES}

Bowers, J., \& Schacter, D. L. (1993). Priming of novel information in amnesic patients: Issues and data. In P. Graf \& M. Masson (Eds.), Implicit memory: New directions in cognition, development, and neuropsychology (pp. 303326). Hillsdale, NJ: Erlbaum.

Chapman, L. J., Chapman, J. P., Curran, T., \& Miller, M. B (1994). Do children and the elderly show heightened semantic priming? How to answer the question. Developmental Review, 14, 159-185.

Cleeremans, A. (1993). Mechanisms of implicit learning: Connectionist models of sequence processing. Cambridge, MA: MIT Press.

Cleeremans, A., \& McClelland, J. L. (1991). Learning the structure of event sequences. Journal of Experimental Psycbology: General, 120(3), 235-253.

Cohen, A., Ivry, R. I., \& Keele, S. W. (1990). Attention and structure in sequence learning. Journal of Experimental Psychology: Learning, Memory, and Cognition, 16, 17-30.

Cohen, N. J., \& Eichenbaum, H. (1993). Memory, amnesia, and the bippocampal system. Cambridge: MIT Press.

Cohen, N. J., \& Squire, L. R. (1980). Preserved learning and retention of pattern analysing skill in amnesia: Dissociation of "knowing how" and "knowing that." Science, 210, 207209.

Curran, T. (1995). On the neural mechanisms of sequence learning. Psyche, 2(12), URL:http://psyche.cs.monash. edu.au/volume2-1/psyche-95-2-12-sequence-1-curran.html.

Curran, T. (1997). Effects of aging on implicit sequence learning: Accounting for sequence structure and explicit knowledge. Psychological Research/Psycbologische Forschung, in press.

Curran, T., \& Schacter, D. L. (in press). Implicit memory: What must theories of amnesia explain? Memory.

Elman, J. L. (1990). Finding structure in time. Cognitive Science, 14, 117-211.

Gabrieli, J. (1994). Contributions of the basal ganglia to skill learning and working memory in humans. In J. Houk, J. L. Davis, \& D. G. Beiser (Eds.), Information processing in the basal ganglia (pp. 277-294). Cambridge, MA: MIT Press.

Gluck, M. A., \& Myers, C. E. (1992). Hippocampal-system function in stimulus representation and generalization: A computational theory. Proceedings of the 14th Annual Conference of the Cognitive Science Society (pp. 390395). Hillsdale: Erlbaum.

Graf, P., \& Schacter, D. L. (1985). Implicit and explicit memory for new associations in normal and amnesic subjects. Journal of Experimental Psychology: Learning. Memory. and Cognition. 11, 501-518.

Grafton, S. T., Hazeltine, E., \& Ivry, R. (1995). Functional map- ping of sequence learning in normal humans. Journal of Cognitive Neuroscience, 7, 497-510.

Helmuth, L., \& Ivry, R. B. (in press). Sequential movements. In M. Jahanshahi \& R. Brown (Eds.), The neuropsychology of movement disorders. Amsterdam: Elsevier Science Publishers.

Jackson, G., \& Jackson, S. (1992). Sequence structure and sequential learning: The evidence from ageing reconsidered. (Technical Report 92-9): Institute of Cognitive and Decision Sciences, University of Oregon.

Jackson, G. M., Jackson, S. R., Harrison, J., Henderson, L., \& Kennard, C. (1995). Serial reaction time learning and Parkinson's disease: Evidence for a procedural learning deficit. Neuropsycbologia. 33, 577-593.

Jordan, M. I. (1990). Motor learning and the degrees of freedom problem. In M. Jeannerod (Ed.), Attention and performance XIII (pp. 796-836). Hillsdale, NJ: Erlbaum.

Keele, S. W., \& Jennings, P. J. (1992). Attention in the representation of sequence: Experiment and theory. Human Movement Studies, 11, 125-138.

Knowlton, B. J., Squire, L. R., \& Gluck, M. A. (1994). Probabilistic classification learning in amnesia. Learning and Memory, 1, 106-120.

Mayes, A. R., \& Downes, J. J. (in press). What do theories of the functional deficit(s) underlying amnesia have to explain? Memory.

Moscovitch, M., Vriezen, E., \& Goshen-Gottstein, Y. (1993). Implicit tests of memory in patients with focal lesions or degenerative brain disorders. In H. Spinnler \& F. Boller (Eds.), Handbook of neuropsycbology (Vol. 8, pp. 133-173). Amsterdam: Elsevier.

Musen, G., \& Squire, L. R. (1993a). Implicit learning of colorword associations using a stroop paradigm. Journal of ExDerimental Psycbologv: Learning. Memory and Cognition, 19, 789-798.

Musen, G., \& Squire, L. R. (1993b). On the implicit learning of novel associations by amnesic patients and normal subjects. Neuropsychology 7, 119-135.

Nissen, M. J., \& Bullemer, P. (1987). Attentional requirements of learning: Evidence from performance measures. Cognitive Psycbology, 19, 1-32.

Nissen, M. J., Knopman, D. S., \& Schacter, D. L. (1987). Neurochemical dissociation of memory systems. Neurology, 37, 789-794.

Nissen, M. J., Willingham, D., \& Hartman, M. (1989). Explicit and implicit remembering: When is learning preserved in amnesia? Neurotsvchologia. 27 341-352.

Parkin, A. J., \& Leng, N. R. C. (1993). Neuropsychology of the amnesic syndrome. Hillsdale, NJ: Lawrence Erlbaum Associates.

Pascual-Leon, A., Grafman, J., \& Hallet, M. (1994). Modulation of cortical motor output maps during development of implicit and explicit knowledge. Science, 263, 1287-1289.

Perruchet, P., \& Amorim, M. (1992). Conscious knowledge and changes in performance in sequence learning: Evidence against dissociation. Journal of Experimental Psychology: Learning Memory and Cognition. 18, 785-800.

Rauch, S. L., Savage, C. R., Brown, H. D., Curran, T., Alpert, N. M., Kendrick, A., Fischman, A. J., \& Kosslyn, S. M. (1995). A PET investigation of implicit and explicit sequence learning. Human Brain Mapping, 3, 271-286.

Reber, P. J., \& Squire, L. R. (1994). Parallel brain systems for learning with and without awareness. Learning $\& \mathrm{Mem}$ ory, 1, 217-229.

Reed, J., \& Johnson, P. (1994). Assessing implicit learning with indirect tests: Determining what is learned about sequence structure. Journal of Experimental Psycbology: Learning, Memory, and Cognition, 20, 585-594. 
Rudy, J. W., \& Sutherland, R. J. (1994). The memory-coherence problem, configural associations, and the hippocampal system. In D. L. Schacter \& E. Tulving (Eds.), Memory systems 1994 (pp. 119-146). Cambridge: MIT Press.

Schacter, D. L., Chiu, C. Y. P., \& Ochsner, K. N. (1993). Implicit memory a selective review. Annual Review of Neuroscience, 16, 159-182.

Shanks, D. R., \& St. John, M. F. (1994). Characteristics of dissociable human learning systems. Bebavioral and Brain Sciences, 17, 367-447.

Shapiro, M. L., \& Olton, D. S. (1994). Hippocampal function and interference. In D. L. Schacter \& E. Tulving (Eds.), Memory systems 1994, (pp. 85-117). Cambridge, MA: MIT Press.

Squire, L. R. (1992). Memory and the hippocampus: A synthesis of findings with rats, monkeys, and humans. Psvcbological Review, 99, 195-231.

Stadler, M. A. (1992). Statistical structure and implicit serial learning. Journal of Experimental Psycbology: Learning, Memory, and Cognition, 18, 318-327.

Stadler, M. A. (1993). Implicit serial learning: Questions inspired by Hebb (1961). Memory \& Cognition, 21, 819827.
Stern, C. E., Corkin, S., Gonzalez, R. G., Guimaraes, A. R., Baker, J. R., Jennings, P. J., Carr, C. A., Sugiura, R. M., Vedantham, V., \& Rosen, B. R. (1996). The hippocampal formation participates in novel picture encoding: Evidence from functional magnetic resonance imaging. Proceedings of the National Academy of Sciences, 933, 8660-8665.

Tulving, E., Markowitsch, H. J., Kapur, S., Habib, R., \& Houle, S. (1994). Novelty encoding networks in the human brain: Positron emission tomography data. Neuroreport, 5, 25252528.

Wicklegren, W. A. (1979). Chunking and consolidation: A theoretical synthesis of semantic networks, configuring in conditioning, S-R versus cognitive learning, normal forgetting, the amnesic syndrome, and the hippocampal arousal system. Psycbological Review, 86, 44-60.

Willingham, D. B., Greenley, D. B., \& Bardona, A. M. (1993). Dissociation in a serial response time task using a recognition measure: Comment on Perruchet and Amorim (1992). Iournal of Experimental Psychology: Learning, Memory. and Cognition. 19, 1424-1430.

Willingham, D. B., \& Koroshetz, W. J. (1993). Evidence for dissociable motor skills in Huntington's disease patients. Psychobiology, 21, 173-182. 